PCPS 68-22

Printed in Great Britain

\title{
CORRIGENDUM
}

\section{The low frequency scalar diffraction by an elliptic disc}

\author{
BY B. D. SLEEMAN
}

Department of Mathematics, The University, Dundee

Proc. Cambridge Philos. Soc. 63 (1967), 1273-1280

(Received 10 July 1969)

The above-named article contains algebraic errors in the terms of certain series, particularly in the expressions for the far field amplitude $f$ and Scattering Cross. section. The correct results together with the corresponding equation numbers of the original paper are listed below.

$$
\begin{aligned}
& \omega_{1}\left(P_{1}\right)=i k L\left\{\frac{u F_{0}^{0}\left(\gamma_{1}\right) \operatorname{sn} \gamma_{1}}{u F_{0}^{0}\left(K+i K^{\prime}\right)}+\frac{u E_{0}^{0}\left(K+i K^{\prime}\right) u F_{0}^{0}\left(\gamma_{1}\right)}{\left\{u E_{0}^{0}(0)\right\}^{2}\left\{u F_{0}^{0}\left(K+i K^{\prime}\right)\right\}^{2}}-\frac{u E_{0}^{0}\left(\gamma_{1}\right)}{\left\{u E_{0}^{0}(0)\right\}^{2} u F_{0}^{0}\left(K+i K^{\prime}\right)}\right\} \\
& \omega_{2}\left(P_{1}\right)=-\frac{k^{3} L^{2}}{u F_{0}^{0}\left(K+i K^{\prime}\right)}\left\{\frac{A_{0,1}^{(u)} u F_{2}^{0}\left(\gamma_{1}\right)}{u E p_{2}^{0}(0,0)} \frac{\lambda^{2}}{\bar{\lambda}-\lambda} \int_{k+i k^{\prime}}^{\gamma_{1}} u F_{0}^{0}(\gamma) u E_{2}^{0}(\gamma) \mathrm{sn}^{2} \gamma d \gamma\right. \\
& -\frac{A_{1,1}^{(u)} u F_{2}^{1}\left(\gamma_{1}\right)}{u E p_{2}^{1}(0,0)} \frac{\bar{\lambda}^{2}}{\bar{\lambda}-\lambda} \int_{K+i K^{\prime}}^{\gamma_{1}} u F_{0}^{0}(\gamma) u E_{2}^{1}(\gamma) \operatorname{sn}^{2} \gamma d \gamma \\
& +\frac{A_{0,1}^{(u)} u E_{2}^{0}\left(\gamma_{1}\right)}{u E p_{2}^{0}(0,0)} \frac{\lambda^{2}}{\bar{\lambda}-\lambda} \int_{\gamma_{1}}^{i K^{\prime}} u F_{0}^{0}(\gamma) u F_{2}^{0}(\gamma) \operatorname{sn}^{2} \gamma d \gamma \\
& \left.-\frac{A_{1,1}^{(u)} u E_{2}^{1}\left(\gamma_{1}\right)}{u E p_{2}^{1}(0,0)} \frac{\bar{\lambda}^{2}}{\lambda-\bar{\lambda}} \int_{\gamma_{1}}^{i K^{\prime}} u F_{0}^{0}(\gamma) u F_{2}^{1}(\gamma) \operatorname{sn}^{2} \gamma d \gamma\right\} \\
& +k^{3} L^{2}\left\{A_{0,1}^{(u)} \frac{u F_{2}^{0}\left(\gamma_{1}\right)}{u E p_{2}^{0}(0,0)} \frac{\lambda^{2} \bar{\lambda}}{\lambda-\bar{\lambda}} \int_{K+i K^{\prime}}^{\gamma_{1}} u E_{2}^{0}(\gamma) u F_{0}^{0}(\gamma) d \gamma\right. \\
& -A_{1,1}^{(u)} \frac{u F_{2}^{1}\left(\gamma_{1}\right)}{u \bar{E} p_{2}^{1}(0,0)} \frac{\lambda \bar{\lambda}^{2}}{\bar{\lambda}-\lambda} \int_{K+i K^{\prime}}^{\gamma_{1}} u E_{2}^{1}(\gamma) u F_{0}^{0}(\gamma) d \gamma \\
& +A_{0,1}^{(u)} \frac{u E_{2}^{0}\left(\gamma_{1}\right)}{u E p_{2}^{0}(0,0)} \frac{\lambda^{2} \bar{\lambda}}{\bar{\lambda}-\lambda} \int_{\gamma_{1}}^{i K^{\prime}} u F_{2}^{0}(\gamma) u F_{0}^{0}(\gamma) d \gamma \\
& \left.-A_{1,1}^{(u)} \frac{u E_{2}^{1}\left(\gamma_{1}\right)}{u E p_{2}^{1}(0,0)} \frac{\lambda \bar{\lambda}^{2}}{\bar{\lambda}-\lambda} \int_{\gamma_{1}}^{i K^{\prime}} u F_{2}^{1}(\gamma) u F_{0}^{0}(\gamma) d \gamma\right\} \\
& +\frac{k L^{2} A_{0,0}^{(u)}}{u E p_{0}^{0}(0,0)}\left\{\frac{2}{3} \frac{u F_{0}^{0}\left(\gamma_{1}\right) u F_{0}^{\prime 0}\left(\gamma_{1}\right) u E_{0}^{0}\left(\gamma_{1}\right)}{u F_{0}^{0}\left(K+i K^{\prime}\right)} \operatorname{sn}^{2} \gamma_{1}\right. \\
& -\frac{2}{3 k^{2}} \frac{u E_{0}^{0}\left(K+i K^{\prime}\right) u F_{0}^{\prime 0}\left(K+i K^{\prime}\right) u F_{0}^{0}\left(\gamma_{1}\right)}{u F_{0}^{0}\left(k+i K^{\prime}\right)}
\end{aligned}
$$




$$
\begin{aligned}
& +\frac{u F_{0}^{0}\left(\gamma_{1}\right) u F_{0}^{\prime 0}\left(\gamma_{1}\right) \operatorname{sn} \gamma_{1}}{\left\{u F_{0}^{0}\left(K+i K^{\prime}\right)\right\}^{2}}-\frac{u F_{0}^{\prime 0}\left(\gamma_{1}\right) \operatorname{sn} \gamma_{1}}{u F_{0}^{0}\left(K+i K^{\prime}\right)}-\frac{k}{\left\{u E_{0}^{0}(0)\right\}^{2}\left\{u F_{0}^{0}\left(K+i K^{\prime}\right)\right\}^{2}} \\
& +\frac{k u F_{0}^{0}\left(\gamma_{1}\right)}{\left\{u F_{0}^{0}\left(K+K^{\prime}\right)\right\}^{3}\left\{u E_{0}^{0}(0)\right\}^{2}}-\frac{1}{3 k^{2}} \frac{u E_{0}^{0}\left(\gamma_{1}\right)\left\{u F_{0}^{\prime 0}\left(\gamma_{1}\right)\right\}^{2}\left\{\gamma_{1}-E\left(\gamma_{1}\right)\right\}}{u F_{0}^{0}\left(K+i K^{\prime}\right)} \\
& \left.+\frac{1}{3} u E_{0}^{0}\left(K+i K^{\prime}\right) \frac{\left\{u F_{0}^{\prime 0}\left(\gamma_{1}\right)\right\}^{2}}{k^{2}\left\{u F_{0}^{0}\left(K+i K^{\prime}\right)\right\}^{2}} u F_{0}^{0}\left(\gamma_{1}\right)\left\{K+i K^{\prime}-E\left(K+i K^{\prime}\right)\right\}\right\} \\
& +\frac{L^{2}}{2}\left\{\frac{u E p_{0}^{0}\left(\alpha_{1}, \beta_{1}\right) u F_{0}^{0}\left(\gamma_{1}\right)}{u E p_{0}^{0}(0,0) u F_{0}^{0}\left(K+i K^{\prime}\right)}\left[\left(1-k^{2} m^{2}\right)+k^{2}\left(l^{2}-m^{2}\right) \lambda \bar{\lambda}+k^{2} m^{2}(\lambda+\bar{\lambda})\right]\right\} .
\end{aligned}
$$

$$
\begin{aligned}
f_{s}=\mid \chi k L[ & -\frac{1}{u E_{0}^{0}(0) u F_{0}^{0}\left(K+i K^{\prime}\right)}+\frac{i \chi k L}{\left\{u E_{0}^{0}(0)\right\}^{2}\left\{u F_{0}^{0}\left(K+i K^{\prime}\right)\right\}^{2}} \\
& +\frac{k^{2} L^{2} \chi^{2}}{u F_{0}^{0}\left(K+i K^{\prime}\right)}\left\{\frac{u E p_{2}^{0}(\alpha, \beta)}{u E_{0}^{0}(0) u E p_{2}^{0}(0,0)} \frac{\lambda^{2}}{6(\bar{\lambda}-\lambda)}-\frac{u E p_{2}^{1}(\alpha, \beta)}{u E_{0}^{0}(0) u E p_{2}^{1}(0,0)} \frac{\bar{\lambda}^{2}}{6(\bar{\lambda}-\lambda)}\right. \\
& +\frac{u E p_{0}^{0}(\alpha, \beta)}{u E p_{0}^{0}(0,0)}\left(\frac{1}{\left\{u F_{0}^{0}\left(K+i K^{\prime}\right)\right\}^{2}\left\{u E_{0}^{0}(0)\right\}^{3}}-\frac{\left(1+k^{2}\right)}{18 k^{2} u E_{0}^{0}(0)}+\frac{K-E-i E^{\prime}}{3 k\left\{u E_{0}^{0}(0)\right\}^{2} u \bar{F}_{0}^{0}\left(K+i \overline{K^{\prime}}\right)}\right. \\
& \left.\left.\left.+\frac{1}{2 k u E_{0}^{0}(0)}\left[k^{2}\left(l^{2}-m^{2}\right) \lambda \bar{\lambda}+k^{2} m^{2}(\lambda+\bar{\lambda})-k^{2} m^{2}-\frac{1}{3}\right]\right)\right\}+O\left(\chi^{3}\right)\right] . \\
& \sigma=\frac{4 \pi L^{2}}{K^{2}}\left[1+\chi^{2} L^{2}\left\{\frac{1+k^{2}}{9}-\frac{2}{3} \frac{K-E}{K}\right.\right. \\
& \left.\left.-\frac{1}{K^{2}}-\left[k^{2}\left(l^{2}-m^{2}\right) \lambda \bar{\lambda}+k^{2} m^{2}(\lambda+\bar{\lambda}-1)-\frac{1}{3}\right]\right\}+O\left(\chi^{4}\right)\right] .
\end{aligned}
$$

The first paragraph of the subsequent conclusion becomes redundant and should be replaced by the following.

As a check on the validity of the result $(5 \cdot 6)$ we reduce the elliptic disc to a circular one. This is simply achieved by letting $k \rightarrow 0$. Thus in this limiting case we obtain, on putting either

or

$$
\begin{gathered}
l=\sin \gamma, \quad m=0, \quad n=\cos \gamma, \\
l=0, \quad m=\sin \gamma, \quad n=\cos \gamma, \\
\sigma=\frac{16 L^{2}}{\pi}\left[1+\chi^{2} L^{2}\left(\frac{\pi^{2}-36+3 \pi^{2} \cos ^{2} \gamma}{9 \pi^{2}}\right)+O\left(\chi^{4}\right)\right] .
\end{gathered}
$$

This result is seen to agree with that given by Bazer and Hochstadt (1). Further we remark that $(5 \cdot 6)$ is also in agreement with the corresponding result given by Williams (2), who has considered the same problem using a variational method.

The author is indebted to Professor W. E. Williams for bringing his work to the author's attention.

\section{REFERENCES}

(1) Bazer, B. and Hochstadt, H. Comm. Pure Appl. Math. 15 (1962), 1-33.

(2) Williams, W. E. To be published (1970). 